\title{
Receptor conversion in metastatic breast cancer: a prognosticator of survival
}

\author{
Xiangying Meng ${ }^{1}$, Santai Song ${ }^{2}$, Ze-Fei Jiang ${ }^{2}$, Bing Sun ${ }^{1}$, Tao Wang ${ }^{2}$, Shaohua \\ Zhang', Shikai Wu ${ }^{1}$ \\ ${ }^{1}$ Radiotherapy Department, Affiliated Hospital of Academy of Military Medical Sciences, Beijing, China \\ ${ }^{2}$ Breast Cancer Department, Affiliated Hospital of Academy of Military Medical Sciences, Beijing, China \\ Correspondence to: Shikai Wu, email: skywu4923@sina.com
}

Keywords: breast cancer, estrogen receptor, progesterone receptor, metastases, survival

Received: June 09, $2016 \quad$ Accepted: September 05, 2016

Published: September 19, 2016

\section{ABSTRACT}

Objective: This retrospective study investigated the association between hormone receptor (HR) conversion and survival in breast cancer patients.

Methods: Estrogen receptor (ER) and progesterone receptor (PR) status (positive or negative) of primary tumors and of paired metastatic sites in 627 breast cancer patients were analyzed by McNemar's test for rates of receptor conversion. A survival analysis was performed using the Kaplan-Meier method, and prognostic factors were assessed using Cox's proportional hazards regression model.

Results: Conversion of ER occurred in 165 (26.31\%) patients, and conversion of PR in 213 (33.97\%; $P<0.001$, both). For 82 patients whose ER and PR were reassessed 2-4 times during metastatic progression, ER and PR re-conversion occurred in $22(26.83 \%)$ and $29(35.36 \%)$, respectively. The change of ER or PR from positive to negative was associated with worse overall survival and post-recurrent survival (log-rank; $P<\mathbf{0 . 0 0 1}$, both). A subgroup analysis of HR-positive patients (i.e., positive $E R$, PR, or both) in primary tumor and HR-negative in metastatic sites showed that patients who accepted both salvage endocrine therapy and chemotherapy had better post-recurrent survival than did those who accepted salvage chemotherapy only (logrank; $P=0.003$ ).

Conclusion: ER and PR status may change several times during metastatic tumor progression. A change of HR from positive to negative was associated with worse survival compared with consistent positivity. Repeated evaluations of HR status are necessary in metastatic breast cancer. Salvage hormonal therapy is still worth trying for patients whose HR status changes from positive to negative.

\section{INTRODUCTION}

The 5-year survival rate of women with breast cancer has been improving steadily for the past 5 years, but metastasis to distant sites still limits survival for patients who have undergone radical surgery $[1,2]$. Approximately one-third of women with early-stage breast cancer develops distant metastasis and suffers a tumorrelated death [3-6].

Endocrine therapy as an adjuvant to prevent and treat metastatic breast cancer is generally well tolerated and efficient. However, the response to endocrine agents such as tamoxifen and aromatase inhibitors, whether partial or complete, is closely dependent on the hormone receptor (HR) status in cancer tissues $[7,8]$. Patients whose primary breast tumors are positive for estrogen and progesterone receptors (ER and PR, respectively) are preferred candidates for salvage hormonal therapy, to attenuate progression of the disease.

In routine clinical practice, options for systemic therapy depend on the characteristics of the primary tumor, determined by routine histopathology, immunohistochemistry (IHC), or molecular analysis. However, the choice of treatment may better rest on features of the metastasized lesions rather than that of the primary tumors, in particular with regard to differences in HR status. Differences between the HR 
status of the primary tumors and metastatic lesions, termed receptor conversion, have been confirmed in $18 \%-54 \%$ of breast cancer patients [9-20]. These results support the necessity for biopsies of metastasized lesions, since the specific HR status of these may alter the choice of therapeutic regimen for these patients $[21,22]$.

ER and PR levels in primary breast cancers may be important indicators. For example, ER and PR positivity were associated with better treatment outcomes [23], and ER and PR negativity with poorer clinical outcomes [24]. There is some indication from retrospective studies that breast cancer cells that have undergone HR conversion may be more aggressive [25, $26]$, but a prospective study reported that a therapeutic regimen based on the receptor status of the metastasis did not contribute a survival benefit [10]. Thus the question remains whether identifying the HR status of metastasized breast cancer would aid the choice of therapy strategy.

The present retrospective study of a large cohort of breast cancer patients, compared the ER and PR statuses of primary breast tumors with that of paired metastatic lesions. In addition, we investigated whether HR conversion influenced the survival of these patients, and the effect of HR conversion on salvage hormonal therapy.

\section{RESULTS}

\section{Patients' clinicopathological characteristics}

Six hundred and twenty-seven women with metastatic breast cancer were eligible and involved in our analysis (Table 1). The median ages of the patients at primary tumor diagnosis and metastasis diagnosis were 44 years (range, 22-79 years) and 48 years (range, 25-80 years), respectively.

In primary breast cancers, the positive rates for ER and PR were $55.18 \%$ and $50.24 \%$, respectively. In accordance with the guidelines of the National Comprehensive Cancer Network, positive HR status was defined as positive signs of ER, PR, or both; and negative HR status was defined as negative signs of both ER and PR. In our study, HR positivity was detected in $62.84 \%$ $(394 / 627)$ of all cases.

The biopsied metastatic sites were the following: soft tissues $(n=473)$, liver $(n=96)$, bone $(n=21)$, lung $(n=14)$, ovary $(n=9)$, pleura $(n=5)$, thyroid gland $(n=$ $3)$, brain $(n=2)$, bladder $(n=1)$, stomach $(\mathrm{n}=1)$, kidney $(\mathrm{n}=1)$, and pancreas $(n=1)$. Soft tissues were the most frequent biopsy site of breast cancer metastasis, perhaps because biopsies of soft tissue are safer than of viscera. The next three most common biopsy sites of metastasis were, in descending order, liver, bone, and lung.

Table 1: Patients' clinicopathological characteristics, $n / \mathrm{N}(\%)$

\begin{tabular}{|c|c|c|c|c|c|}
\hline & & \multicolumn{2}{|c|}{ ER } & \multicolumn{2}{|c|}{ PR } \\
\hline & & Positive & Negative & Positive & Negative \\
\hline \multirow[t]{3}{*}{ Age, years } & $<35$ & $76 / 346$ (21.96) & 43/281 (15.30) & $75 / 315(23.81)$ & $44 / 312(14.10)$ \\
\hline & $35-60$ & $252 / 346(72.83)$ & $211 / 281(75.09)$ & $223 / 315(70.79)$ & $240 / 312(76.92)$ \\
\hline & $>60$ & $18 / 346(5.20)$ & $27 / 281(9.61)$ & $17 / 315(5.40)$ & $28 / 312(8.97)$ \\
\hline \multirow[t]{3}{*}{ Clinical stage } & I & $70 / 346(20.23)$ & $40 / 281(14.23)$ & $61 / 315(19.37)$ & $49 / 312(15.71)$ \\
\hline & II & $236 / 346(68.21)$ & $197 / 281(70.11)$ & $212 / 315(67.30)$ & $221 / 312(70.83)$ \\
\hline & III & $40 / 346(11.56)$ & 44/281 (15.66) & 42/315 (13.33) & $42 / 312(13.46)$ \\
\hline \multirow[t]{2}{*}{ Adjuvant therapy } & Chemotherapy & $317 / 346(91.62)$ & $263 / 281(93.59)$ & $293 / 315(93.02)$ & $287 / 312(91.99)$ \\
\hline & Hormone therapy & $248 / 346(71.68)$ & 65/281 (23.13) & $218 / 315(69.21)$ & $95 / 312(30.45)$ \\
\hline \multirow[t]{3}{*}{ HER2 } & Positive $^{\mathrm{a}}$ & $44 / 346(12.72)$ & $71 / 281(25.27)$ & 47/315 (14.92) & $68 / 312(21.79)$ \\
\hline & Negative $^{\mathrm{b}}$ & $255 / 346(73.70)$ & $171 / 281(60.85)$ & $226 / 315(71.75)$ & $200 / 312(64.10)$ \\
\hline & NA & $47 / 346(13.58)$ & 39/281 (13.88) & $42 / 315(13.33)$ & $44 / 312(14.10)$ \\
\hline \multirow[t]{3}{*}{ Menstrual status } & Menopause & $69 / 346(19.94)$ & 75/281 (26.69) & $56 / 315(17.78)$ & $88 / 312(28.21)$ \\
\hline & Pre-menopause & $257 / 346(74.28)$ & $186 / 281(66.19)$ & $242 / 315(76.83)$ & $201 / 312(64.42)$ \\
\hline & Unknown & $20 / 346(5.78)$ & $20 / 281(7.12)$ & $17 / 315(5.4)$ & $23 / 312(7.37)$ \\
\hline
\end{tabular}

Abbreviations: ER, estrogen receptor; PR, progesterone receptor; HER2, human epidermal growth factor receptor 2; IHC, immunohistochemistry; FISH, fluorescence in situ hybridization; NA, Not Available

a $3+$ by IHC or FISH $(+)$

${ }^{\mathrm{b}} 0-2+$ by IHC or FISH $(-)$ 


\section{HR conversion}

A difference in ER status between the primary breast cancer and metastatic lesions was observed in 165 of 627 patients (26.31\%). Specifically, in 106 of 346 patients $(30.63 \%)$, the ER status had altered from positive to negative, while 59 of 281 patients $(21.00 \%)$ had an alteration from negative to positive (McNemar's test, $P$ $<0.001)$.

A difference in PR status was observed in 213 of 627 cases (33.97\%): in 158 of 315 patients $(50.16 \%)$ the PR status had changed from positive in primary tumors to negative in the metastatic tissues, while 55 of 312 patients $(17.63 \%)$ had changed from negative to positive (McNemar's test, $P<0.001$ ).

Thus, HR status conversion was detected in 170 of 627 cases (27.11\%): 121 of 394 (30.71\%) had changed from HR-positive in primary tumors to HR-negative in the metastatic tissues, and 49 of $233(21.03 \%)$ from HRnegative to HR-positive (McNemar's test, $P<0.001$ ).

Reassessments were made from multiple biopsies from various organs during tumor progression in 82 patients with advanced breast cancers. The re-conversion rates for ER and PR were $26.83 \%$ and $35.36 \%$, respectively (McNemar's test, $P<0.05$ ).

Unlike HR status, evidence of human epidermal growth factor receptor 2 (HER2) reflects a more aggressive cancer. The rate of discordance of HER2 between primary tumors and metastases was lower than that of ER and PR: 33 of $503(6.56 \%)$ patients had HER2-negative primary tumors but HER2-positive metastases, and 22 of 503 (4.37\%) had HER2-positive primary tumors but HER2negative metastases (Table 2).

\section{Survival analysis}

The median follow-up time was 35 months (range, 4-220 months). The median OS (the time from primary breast cancer diagnosis to the date of death or the end of follow-up) and post-recurrent survival (the time from metastatic breast cancer diagnosis to the date of death or the end of follow-up) was calculated on the basis of ER status in the primary tumor and metastatic tissues (Table 3).

According to the ER status of the primary tumor and metastatic lesions, we divided all 627 breast cancer patients into 4 groups, as follows. Group A: ER-positive in primary tumor and ER-positive in metastatic tissues; Group B: ER-positive in primary tumor and ER-negative in metastatic tissues; Group C: ER-negative in primary tumor and ER-positive in metastatic tissues; and Group D: ER-negative in primary tumor and ER-negative in metastatic tissues.

There were significant differences in OS (log-rank, $P<0.001$, Figure 1A) and post-recurrent survival (logrank, $P<0.001$, Figure 1B) among the 4 groups. The median OS was 135 (95\% CI 88-NA) months in Group A,
85 (95\% CI 55-141) months in Group B, 107 (95\% CI 75156) months in Group C, and 73 (95\% CI 42-107) months in Group D. The median post-recurrent survival was 68 (95\% CI 44-NA) months in Group A, 43 (95\% CI 27-90) months in Group B, 56 (95\% CI 41-83) months in Group C, and 39 (95\% CI 28-71) months in Group D.

According to the PR status of the primary tumor and metastatic lesions, we divided all 627 breast cancer patients into 4 groups, as follows. Group A: PR-positive in primary tumor and PR-positive in metastatic tissues; Group B: PR-positive in primary tumor and PR-negative in metastatic tissues; Group C: PR-negative in primary tumor and PR-positive in metastatic tissues; and Group D: PR-negative in primary tumor and PR-negative in metastatic tissues.

There were significant differences in OS (log-rank, $P<0.001$, Figure 2A) and post-recurrent survival (logrank, $P<0.001$, Figure 2B) among the 4 groups. The median OS was 121 (95\% CI 90-NA) months in Group A, 107 (95\% CI 64-NA) months in Group B, 126 (95\% CI 73-156) months in Group C, and 79 (95\% CI 45-142) months in Group D. The median post-recurrent survival was 64 (95\% CI 44-NA) months in Group A, 51 (95\% CI 34-NA) months in Group B, 64 (95\% CI 35-89) months in Group C, and 41 (95\% CI 28-82) months in Group D.

After adjusting for the age of patients with primary breast cancer, age of patients with metastatic breast cancer, PR status in metastatic sites, tumor stage, adjuvant endocrine therapy, adjuvant chemotherapy, and salvage endocrine therapy, the multivariate Cox proportional hazards model was applied. The results of pairwise analysis showed that patients with an ER status that changed from positive in the primary tumor to negative in the metastatic ( $\mathrm{Prim}^{+} / \mathrm{Met}^{-}$group) had a significantly increased hazard ratio for death compared with patients with no change in positive ER status $\left(\mathrm{Prim}^{+} / \mathrm{Met}^{+}\right.$group; hazard ratio: $1.74 ; 95 \%$ confidential interval $[\mathrm{CI}]: 1.19$ $2.56, P=0.005$, Table 4).

\section{Salvage hormonal therapy}

A subgroup analysis of this cohort was conducted to investigate the effects of salvage endocrine therapy on breast cancer patients whose HR status changed from positive to negative (121 patients). Of the 121 breast cancer patients in this group, 76 patients received both salvage endocrine therapy and chemotherapy, and 45 patients received salvage chemotherapy only. Univariate Kaplan-Meier analysis was performed to investigate the response to salvage endocrine therapy in terms of post-recurrent survival. We found that the median postrecurrent survival of patients who accepted both salvage endocrine therapy and chemotherapy (71, 95\% CI 28-90 months) was better than that of the chemotherapy-only group (37, 95\%C I 19-50 months; log rank; $P=0.030$; Figure 3C). 
Table 2: HER2 status of primary tumor/metastases, by HR status of primary tumor/metastases

\begin{tabular}{lccccc}
\hline HR status & b & \multicolumn{5}{c}{ HER2 status } \\
& arim & & \\
& Prim $^{+} \mathbf{M e t}^{+}$ & Prim $^{-} / \mathbf{M e t}^{+}$ & Prim $^{+} / \mathbf{M e t}^{-}$ & Prim $^{-} / \mathbf{M e t}^{-}$ & Sum \\
\hline Prim $^{+} / \mathrm{Met}^{+}$ & $16 / 503(3.18)$ & $14 / 503(2.78)$ & $10 / 503(1.99)$ & $184 / 503(36.58)$ & 224 \\
Prim $^{-} /$Met $^{+}$ & $4 / 503(0.80)$ & $1 / 503(0.20)$ & $1 / 503(0.20)$ & $31 / 503(6.16)$ & 37 \\
Prim $^{+} /$Met $^{-}$ & $16 / 503(3.18)$ & $8 / 503(1.59)$ & $4 / 503(0.80)$ & $67 / 503(13.32)$ & 95 \\
Prim $^{-} /$Met $^{-}$ & $39 / 503(7.75)$ & $10 / 503(1.99)$ & $7 / 503(1.39)$ & $91 / 503(18.09)$ & 147 \\
\hline Sum & 75 & 33 & 22 & 373 & 503 \\
\hline
\end{tabular}

Abbreviations: HR, hormone receptor; HER2, human epidermal growth factor receptor 2; Prim, receptor status of the primary lesion; Met, receptor status of the metastatic lesion.

${ }^{a}$ HER2 positive: $3+$ by IHC or FISH (+); HER2 negative: $0-2+$ by IHC or FISH (-)

${ }^{\mathrm{b}} \mathrm{HR}$ positive: ER and/or PR positive; HR negative: ER and PR negative.

Table 3: Effects of HR status in primary tumors and metastatic lesions on OS, median months (95\% CI)

\begin{tabular}{|c|c|c|c|c|c|c|c|}
\hline & & $n$ & Deaths & $\mathrm{OS}^{\mathrm{a}}$ & $P$ & $\mathrm{OS}^{\mathrm{b}}$ & $P$ \\
\hline \multirow[t]{4}{*}{ ER } & $\mathrm{Prim}^{+} / \mathrm{Met}^{+}$ & 240 & 69 & 135(88-NA) & $<0.001^{*}$ & 68 (44-NA) & $<0.001^{*}$ \\
\hline & $\operatorname{Prim}^{-} / \mathrm{Met}^{+}$ & 59 & 27 & $85(55-141)$ & $0.125^{* *}$ & $43(27-90)$ & $0.142^{* *}$ \\
\hline & $\operatorname{Prim}^{+} / \mathrm{Met}^{-}$ & 106 & 45 & $107(75-156)$ & $<0.001^{* *}$ & $56(41-83)$ & $0.001^{* *}$ \\
\hline & $\mathrm{Prim}^{-} / \mathrm{Met}^{-}$ & 222 & 83 & $73(42-107)$ & $<0.001^{* *}$ & $39(28-71)$ & $<0.001^{* *}$ \\
\hline \multirow[t]{4}{*}{ PR } & $\operatorname{Prim}^{+} / \mathrm{Met}^{+}$ & 157 & 48 & 121 (90-NA) & $<0.001^{*}$ & 64 (44-NA) & $<0.001^{*}$ \\
\hline & $\mathrm{Prim}^{-} / \mathrm{Met}^{+}$ & 55 & 26 & 107 (64-NA) & $0.608^{* *}$ & $51(34-N A)$ & $0.212^{* *}$ \\
\hline & $\mathrm{Prim}^{+} / \mathrm{Met}^{-}$ & 158 & 59 & $126(73-156)$ & $0.133^{* *}$ & $64(35-89)$ & $0.086^{* *}$ \\
\hline & $\mathrm{Prim}^{-} / \mathrm{Met}^{-}$ & 257 & 91 & $79(45-142)$ & $<0.001^{* *}$ & $41(28-82)$ & $<0.001^{* *}$ \\
\hline \multirow[t]{4}{*}{$\mathrm{HR}^{\mathrm{c}}$} & $\mathrm{Prim}^{+} / \mathrm{Met}^{+}$ & 273 & 86 & 126 (86-NA) & $<0.001^{*}$ & 64 (43-NA) & $<0.001^{*}$ \\
\hline & $\mathrm{Prim}^{-} / \mathrm{Met}^{+}$ & 49 & 24 & $105(62-156)$ & $0.091^{* *}$ & $56(34-83)$ & $0.095^{* *}$ \\
\hline & $\mathrm{Prim}^{+} / \mathrm{Met}^{-}$ & 121 & 50 & $85(55-122)$ & $<0.001^{* *}$ & $44(27-90)$ & $0.002^{* *}$ \\
\hline & Prim $^{-} / \mathrm{Met}^{-}$ & 184 & 64 & $73(42-111)$ & $<0.001^{* *}$ & $39(28-82)$ & $<0.001^{* *}$ \\
\hline
\end{tabular}

Abbreviations: ER, estrogen receptor; PR, progesterone receptor; Prim, receptor status of the primary lesion; Met, receptor status of the metastatic lesion; NA, not available; $n$, number of patients.

${ }^{\text {a }}$ From breast cancer diagnosis to death or censoring.

${ }^{\mathrm{b}}$ From breast cancer metastases to death or censoring.

${ }^{c}$ HR positive: ER, PR, or both positive; HR negative: ER and PR both negative.

* Compared among 4 groups.

** Compare with Prim ${ }^{+} / \mathrm{Met}^{+}$group.

However, in the $\mathrm{Prim}^{-} / \mathrm{Met}^{-}$group, the postrecurrent survival of the patients who accepted both salvage chemotherapy and endocrine therapy $(n=32)$ was comparable with the patients who accepted chemotherapy only ( $n=152$; log rank test, $P=0.266$; Figure $3-D)$. In the Prim ${ }^{+} \mathrm{Met}^{+}$(Figure 3-A) or Prim $/ \mathrm{Met}^{+}$(Figure 3-B) groups, the post-recurrent survival of the patients who accepted both salvage chemotherapy and endocrine therapy was also comparable with the patients who accepted chemotherapy only. However, it is worth noting that most patients with positive HR accepted endocrine therapy. Therefore, the sample size of the chemotherapyonly subgroup was very small ( 8 patients in the Prim ${ }^{-} / \mathrm{Met}^{+}$ group and 23 patients in $\mathrm{Prim}^{+} / \mathrm{Met}^{+}$group). The small sample size restricts the power of these two subgroups to convince.

The multivariate Cox proportional hazards model was applied. After adjusting for the age of patients with primary breast cancer, age of patients with metastatic breast cancer, tumor stage, adjuvant endocrine therapy, adjuvant chemotherapy, clinical phase, and metastatic sites, the results showed that patients with salvage 

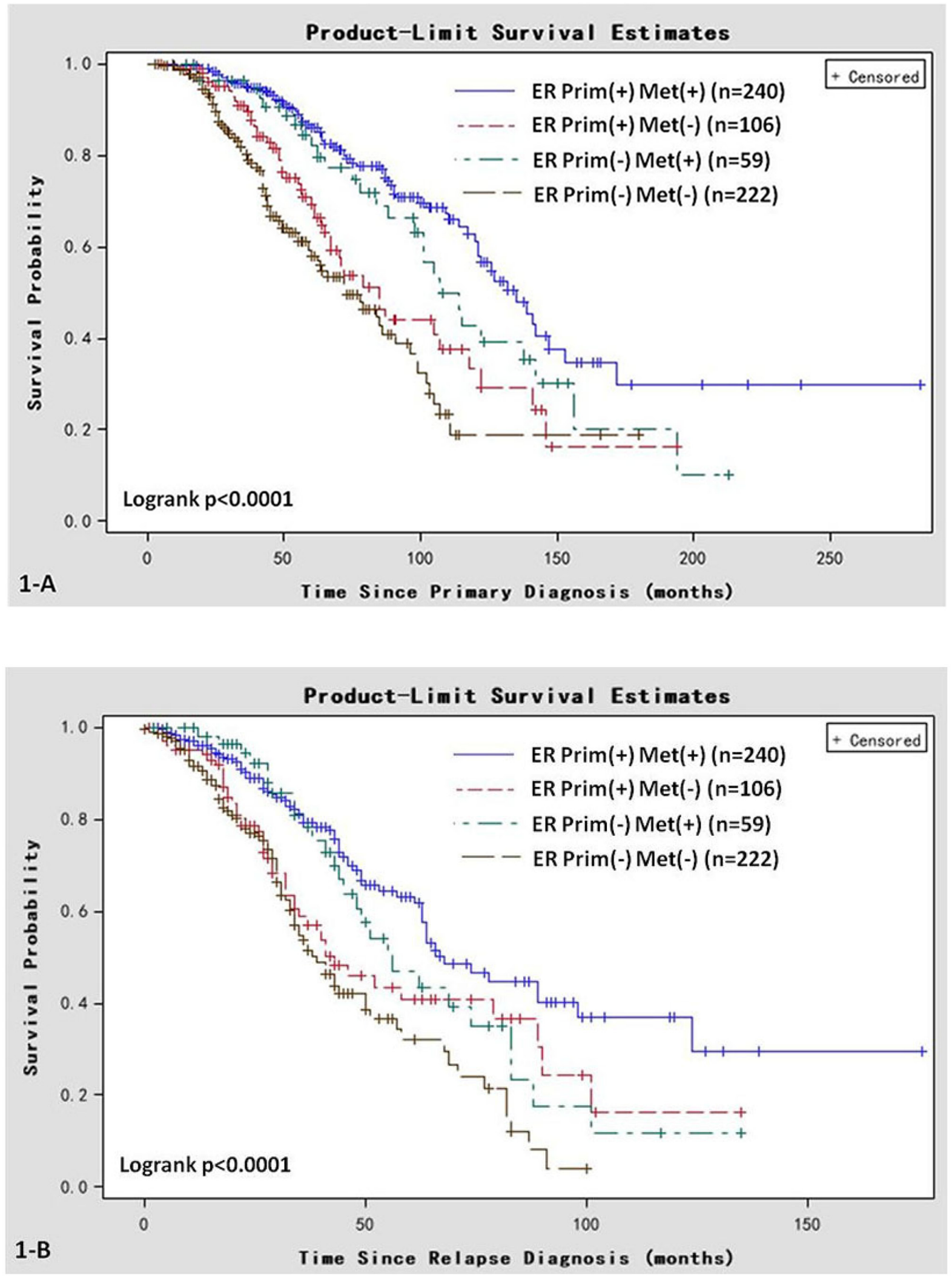

Figure 1: Kaplan-Meier survival curves in women of various ER status subtypes. A. OS associated with various ER statuses in primary breast cancer (Prim) or metastatic sites (Met). B. Post-recurrent survival associated with various ER statuses in primary breast cancer (Prim) and metastatic sites (Met). 

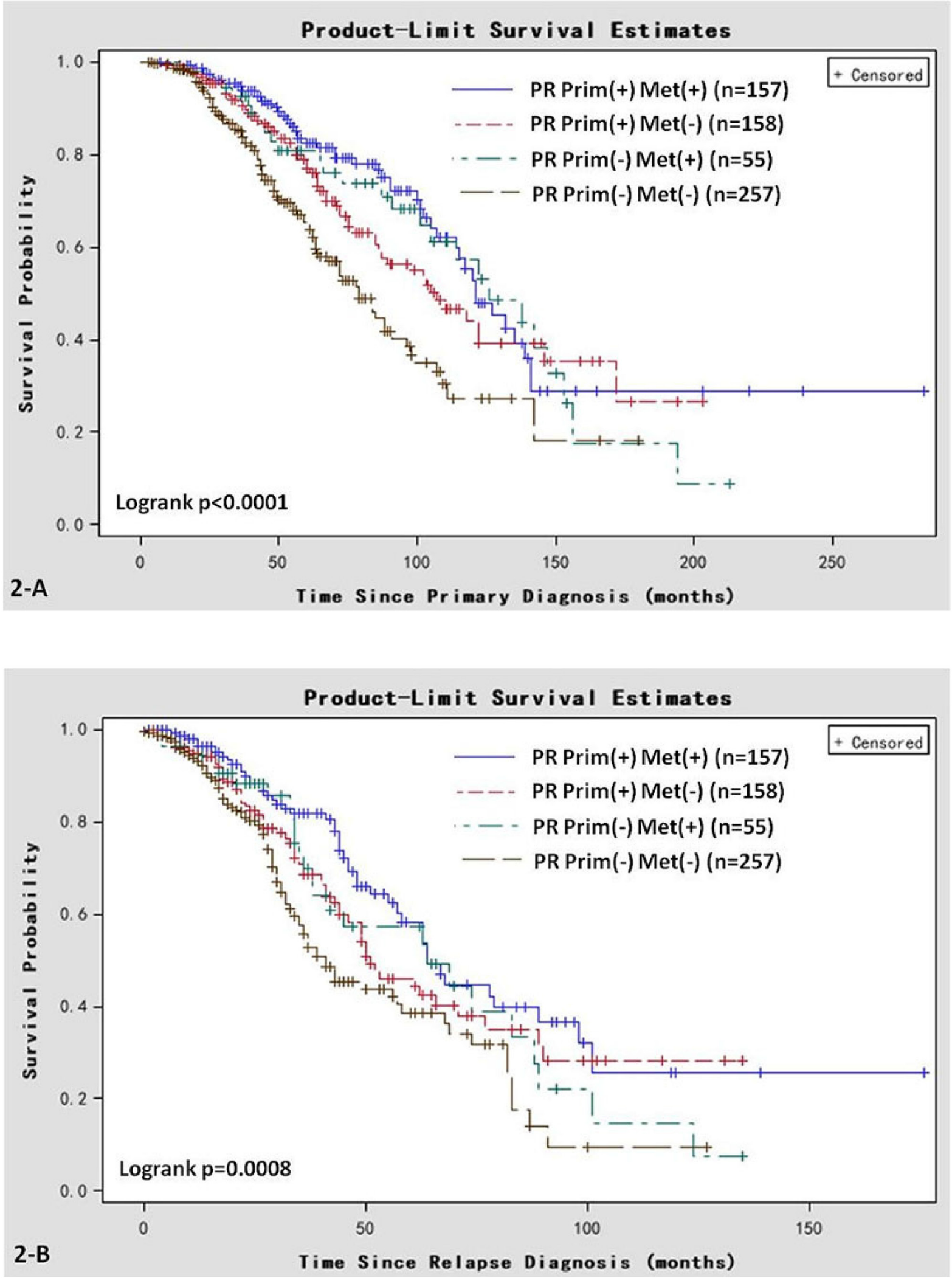

Figure 2: Kaplan-Meier survival curves in women of various PR status subtypes. A. OS associated with various PR statuses in primary breast cancer (Prim) and/or metastatic sites (Met). B. Post-recurrent survival associated with various PR statuses in primary breast cancer (Prim) and metastatic sites (Met). 
Table 4: Risks for patients with breast cancer, depending on ER status in primary tumor and metastases

\begin{tabular}{|c|c|c|c|c|c|c|c|c|c|c|c|}
\hline & & & & \multicolumn{2}{|r|}{$\mathrm{OS}^{\mathrm{a}}$} & \multicolumn{2}{|c|}{ Trend test } & \multicolumn{2}{|c|}{$\mathrm{OS}^{\mathrm{b}}$} & \multicolumn{2}{|c|}{ Trend test } \\
\hline & & \multicolumn{2}{|c|}{ Deaths, $n$} & $\mathrm{HR}^{\mathrm{c}}$ & $95 \% \mathrm{CI}$ & $P$ & $\chi^{2}$ & $\mathrm{HR}^{\mathrm{c}}$ & $95 \% \mathrm{CI}$ & $P$ & $\chi^{2}$ \\
\hline \multirow[t]{4}{*}{ ER } & $\begin{array}{c}\operatorname{Prim}^{+} / \\
\mathrm{Met}^{+}\end{array}$ & 240 & 69 & $1.0^{\mathrm{d}}$ & - & $<0.001^{*}$ & 20.81 & $1.0^{\mathrm{d}}$ & - & $0.004^{*}$ & 13.61 \\
\hline & $\begin{array}{c}\text { Prim }^{-} / \\
\mathrm{Met}^{+}\end{array}$ & 59 & 27 & 1.28 & 0.81 to 2.01 & $0.287^{* *}$ & & 1.18 & 0.75 to 1.86 & $0.470^{* *}$ & \\
\hline & $\begin{array}{c}\operatorname{Prim}^{+} / \\
\mathrm{Met}^{-}\end{array}$ & 106 & 45 & 1.74 & 1.19 to 2.56 & $0.005^{* *}$ & & 1.66 & 1.13 to 2.44 & $<0.001^{* *}$ & \\
\hline & $\begin{array}{c}\operatorname{Prim}^{-} / \\
\mathrm{Met}^{-}\end{array}$ & 222 & 83 & 2.16 & 1.53 to 3.05 & $\underset{* *}{<0.001}$ & & 1.80 & 1.28 to 2.53 & $<0.001^{* *}$ & \\
\hline \multirow[t]{4}{*}{ HR } & $\begin{array}{c}\mathrm{Prim}^{+} / \\
\mathrm{Met}^{+}\end{array}$ & 181 & 72 & $1.0^{\mathrm{d}}$ & - & $0.001^{*}$ & 20.72 & $1.0^{\mathrm{d}}$ & - & $0.007^{*}$ & 12.06 \\
\hline & $\begin{array}{c}\text { Prim }^{-} / \\
\mathrm{Met}^{+}\end{array}$ & 32 & 19 & 1.37 & 0.86 to 2.18 & $0.192^{* *}$ & & 1.22 & 0.77 to 1.93 & $0.404^{* *}$ & \\
\hline & $\begin{array}{c}\mathrm{Prim}^{+} / \\
\mathrm{Met}^{-}\end{array}$ & 102 & 52 & 1.58 & 1.10 to 2.27 & $0.013^{* *}$ & & 1.48 & 1.03 to 2.11 & $0.033^{* *}$ & \\
\hline & $\begin{array}{c}\text { Prim }^{-} / \\
\mathrm{Met}^{-}\end{array}$ & 125 & 66 & 2.21 & 1.56 to 3.13 & $\underset{* *}{<0.001}$ & & 1.78 & 1.27 to 2.50 & $<0.001^{* *}$ & \\
\hline
\end{tabular}

Abbreviations: ER, estrogen receptor; PR, progesterone receptor; HR, hazard ratio; OS, overall survival; Prim, receptor status of the primary lesion; Met, receptor status of the metastatic lesion; No., number of patients.

Adjusted for age and calendar year of primary breast cancer diagnosis, relapse diagnosis, progesterone receptor status, tumor stage, hormonal treatment, and chemotherapy.

${ }^{\text {a }}$ From breast cancer diagnosis to death or censoring.

${ }^{\mathrm{b}}$ From breast cancer relapse to death or censoring.

${ }^{c}$ HR positive: ER and/or PR positive; HR negative: ER and PR negative

d Reference

${ }^{*}$ Compare between 4 groups.

** Compare with $\mathrm{Prim}^{+} / \mathrm{Met}^{+}$group.

chemotherapy-only had a significantly higher hazard ratio for death compared with patients with both salvage endocrine therapy and chemotherapy (hazard ratio: $1.90 ; 95 \%$ confidential interval [CI]: 1.01-3.58, $P=0.045)$.

With regard to adjuvant endocrine therapy, the age of patients with metastatic breast cancer, tumor stage, adjuvant chemotherapy, clinical phase, and metastatic sites showed no statistically significant association with post-recurrent survival in this multivariate Cox proportional hazards model (Table 5).

Univariate Kaplan-Meier analyses of other parameters were also performed. In the $\mathrm{Prim}^{+} / \mathrm{Met}^{-}$ group, the post-recurrent survival of the patients who had accepted adjuvant endocrine therapy was comparable with that of patients who did not accept adjuvant endocrine therapy ( $\log \operatorname{rank}, P=0.194$, Figure 4-A). The post-recurrent survival of patients $\leq 35$ years old at diagnosis of the primary tumor was comparable to that of patients aged 35-60 years or $>60$ years (log rank,
$P=0.567$, Figure 4-B). The post-recurrent survival of patients aged $\leq 35$ years at diagnosis of the metastatic tumor was comparable to that of patients aged 35-60 years old or $>60$ years (log rank, $P=0.523$, Figure 4-C). The post-recurrent survival of the patients who had accepted adjuvant chemotherapy was comparable to that of patients who did not receive adjuvant chemotherapy (log rank, $P=0.590$, Figure 4-D). The differences among the 4 groups of metastatic sites (i.e., distant lymph node metastases; bone metastases; local relapse; and visceral metastases) was not statistically significant (log rank, $P$ $=0.102$, Figure 4-E). The differences among the clinical phase 1, 2, and 3 groups was not statistically significant (log rank, $P=0.409$, Figure 4-F).

\section{DISCUSSION}

In this retrospective clinical study, we compared the ER and PR status of primary breast tumors with matched metastases of 627 breast cancer patients, 

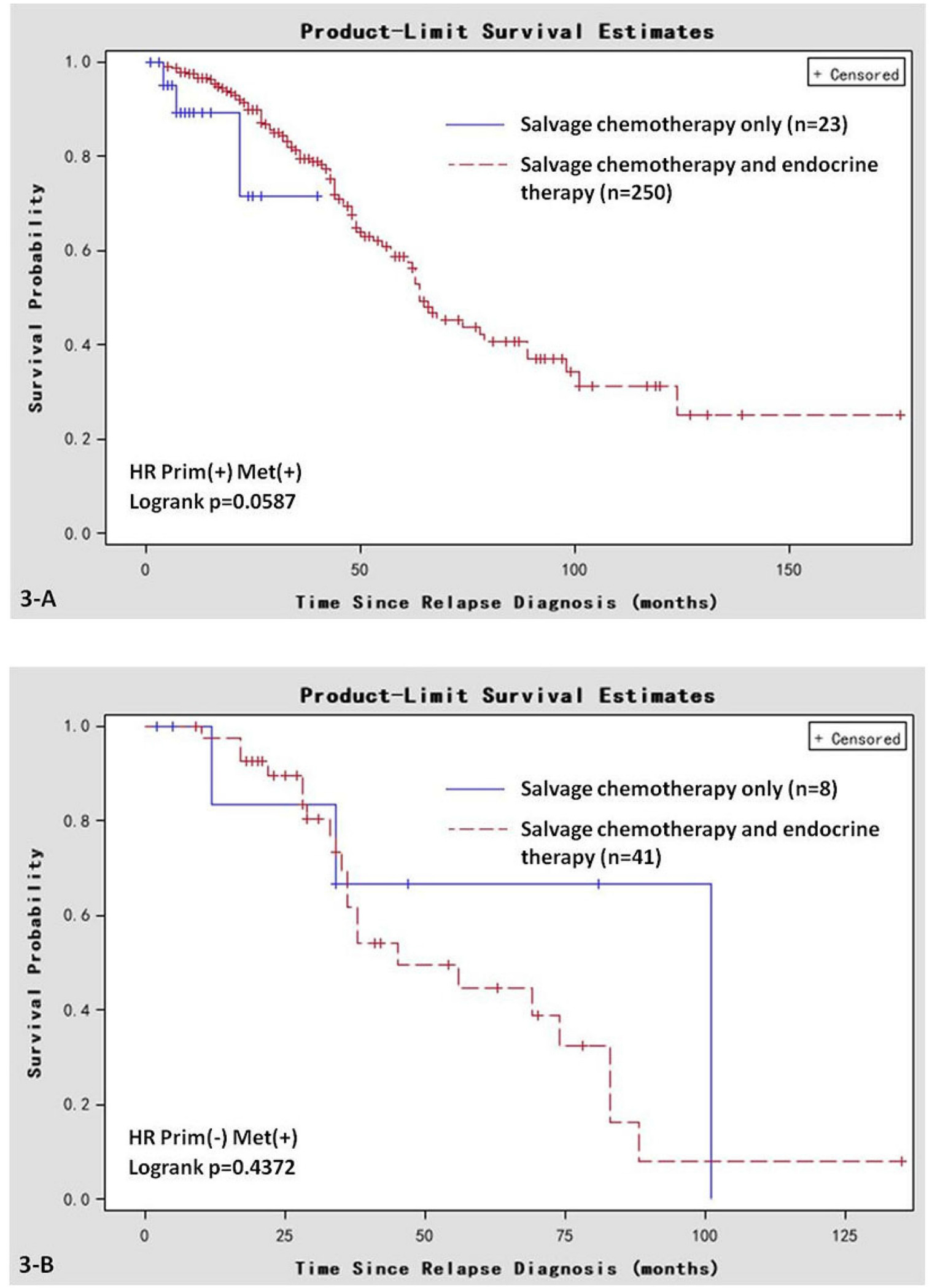

Figure 3: Kaplan-Meier survival curves in women that are HR positive in primary breast cancer and negative in metastatic sites. Post-recurrent survival of the cohort receiving both salvage chemotherapy and endocrine therapy, or salvage chemotherapy only. A. Prim $+\mathrm{Met}^{+}$group; B. Prim $/ \mathrm{Met}^{+}$group. (Continued) 

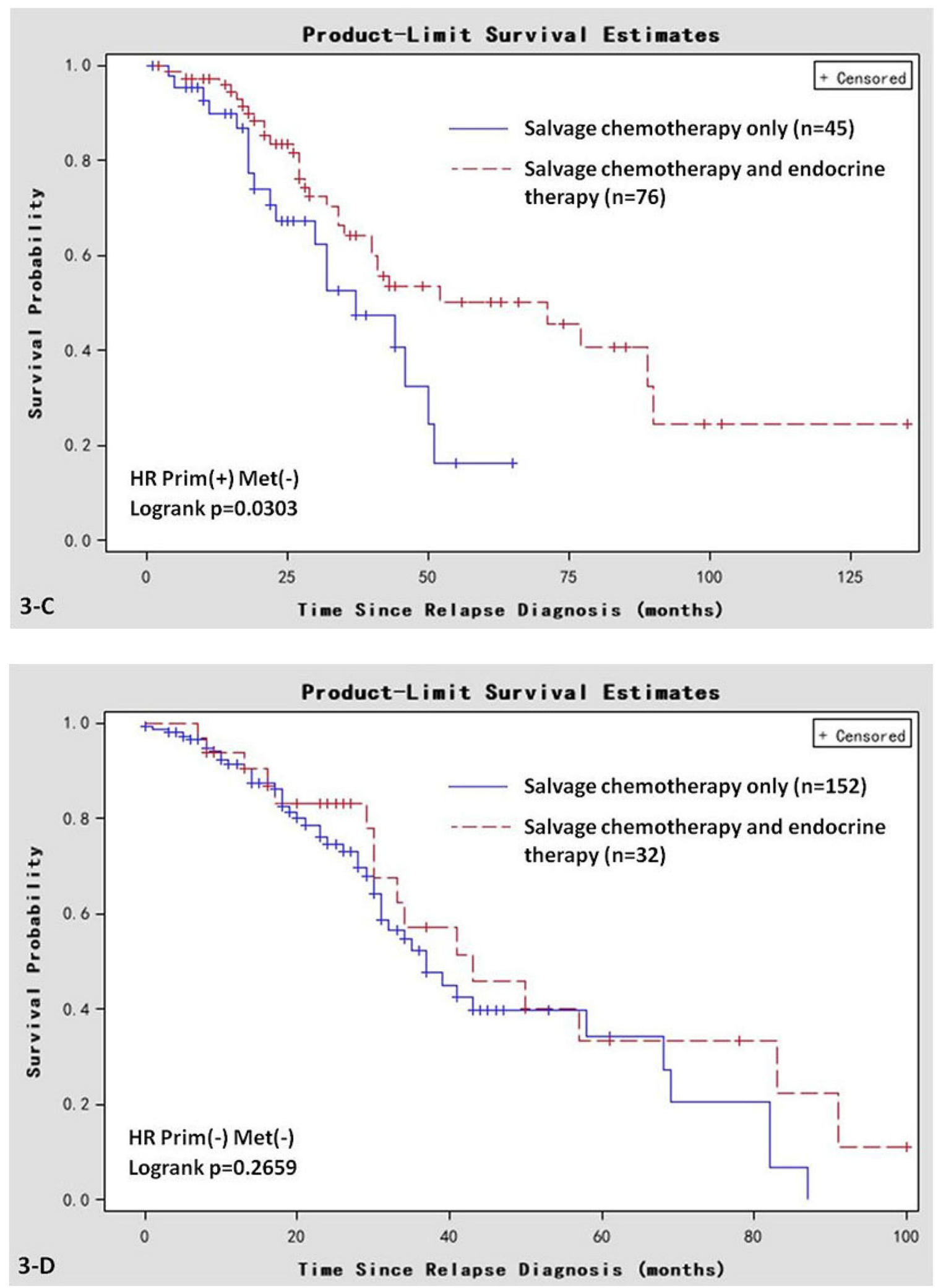

Figure 3: (Continued) Kaplan-Meier survival curves in women that are HR positive in primary breast cancer and negative in metastatic sites. C. $\mathrm{Prim}^{+} / \mathrm{Met}^{-}$group; D. Prim ${ }^{-} \mathrm{Met}^{-}$group. 
Table 5: Multivariate Cox proportional hazards model for post-recurrent survival in $121 \mathrm{Prim}^{+} / \mathrm{Met}^{-}$patients

\begin{tabular}{lcccccc}
\hline & $\begin{array}{c}\text { Parameter } \\
\text { estimate }\end{array}$ & Standard error & $\chi^{2}$ & $\mathbf{P}^{*}$ & Hazard ratio & 95\% CI \\
\hline $\begin{array}{l}\text { Salvage endocrine therapy } \\
\text { Adjuvant endocrine }\end{array}$ & 0.643 & 0.322 & 3.984 & 0.045 & 1.902 & 1.012 to 3.577 \\
therapy & -0.399 & 0.297 & 1.805 & 0.179 & 0.671 & 0.375 to 1.201 \\
Metastatic sites & 0.118 & 0.111 & 1.129 & 0.290 & 1.126 & 0.905 to 1.400 \\
\hline Adjuvant chemotherapy & -0.732 & 0.561 & 1.704 & 0.192 & 0.481 & 0.160 to 1.444 \\
Age at primary diagnosis & 0.087 & 0.078 & 1.222 & 0.269 & 1.091 & 0.935 to 1.272 \\
\hline Age at relapse diagnosis & -0.101 & 0.078 & 1.670 & 0.196 & 0.904 & 0.775 to 1.054 \\
Clinical phase & -0.288 & 0.203 & 2.005 & 0.158 & 0.750 & 0.504 to 1.117 \\
\hline
\end{tabular}

and found evidence that the ER or PR status may be different between primary tumor and metastatic tissues. Furthermore, in some metastatic sites, as the disease progresses, the ER and PR status may change again. We also observed that an ER or PR conversion from positive to negative was negatively associated with OS and post-recurrent survival in these patients. A subgroup analysis of patients with HR-positive status (ER, PR, or both positive) in the primary tumor but HR-negative (ER and PR both negative) in metastatic sites showed that patients who accepted both salvage endocrine therapy and chemotherapy had better post-recurrent survival than did those who accepted chemotherapy only. Systemic therapy prolonged the survival of patients with metastatic breast cancer. HR status in metastatic lesions that differs from that of the primary tumor may have clinical implications in salvage therapy and management.

Several studies have shown that ER and PR were not stable during carcinogenesis and tumor progression $[12,16,21,25,27-38]$. In our study, we investigated the conversion of ER and PR in a large cohort of women with breast cancer metastasis. The inclusion and exclusion criteria were chosen to omit bias. We found that the rates of receptor conversion were similar to several other retrospective studies [21, 36, 39]. It is likely that ER and PR conversion results from genetic mutation during tumor progression, intratumoral heterogeneity, and the selective pressure of therapies [29]. A suitable prospective study is needed to determine better the rates of receptor conversion.

According to previous studies, ER and PR conversion in both primary and metastatic lesions in metastatic breast cancer was a prognosticator of OS and post-recurrent survival time $[29,32,39,40]$. We hypothesize that this correlation can be ascribed to inappropriate target therapy and selection of tumor cells with an unstable phenotype, which may result in more aggressive behaviors [41]. The results of a subsequent univariate analysis showed that a change from positive to negative ER or PR between the primary tumor and metastatic sites was significantly associated with shorter OS compared with no changes. A multi-factorial analysis revealed that only ER status correlated with OS, which was similar to the results of another retrospective study published previously in a peer-reviewed journal [42].

Prospective clinical trials have shown that $14 \%$ of patients with breast cancer had their therapeutic regimen modified as a result of changes in the HR/HER2 status in primary tumor tissues or metastatic cancerous tissue despite endocrine treatment before biopsy [10]. In our study, we investigated HR conversion in metastatic breast cancer as a prognostic biomarker during salvage hormonal therapy. Thus, it was of interest that patients whose HR status had altered from positive to negative still achieved a survival benefit from endocrine treatment, compared with those who did not receive endocrine agents. It is likely that HR status in breast cancer tissues will undergo changes several times during tumor progression. In this 627-patient cohort, for 82 patients whose ER and PR were reassessed 2-4 times during metastatic progression, ER and PR re-conversion occurred in $22(26.83 \%)$ and $29(35.36 \%)$, respectively. Thus, our study shows that subsequent and repeated evaluations are necessary for the metastatic breast cancer patient, and salvage hormonal therapy should not be abandoned because of a positive-tonegative HR conversion between the primary tumor and metastatic lesion.

Although we gained some useful insights in our study, its retrospective nature restricts its power to convince. Another shortcoming of our study is a relatively small sample size for the subgroup analysis, especially for the subgroup analysis of the salvage endocrine therapy in the $\mathrm{Prim}^{+} / \mathrm{Met}^{-}$group. Therefore, a randomized controlled trial is necessary to verify our findings.

In conclusion, ER and PR conversion does occur in breast cancer metastases, and significantly influences survival. Furthermore, ER and PR status may change during tumor progression. Repeated evaluations of HR 

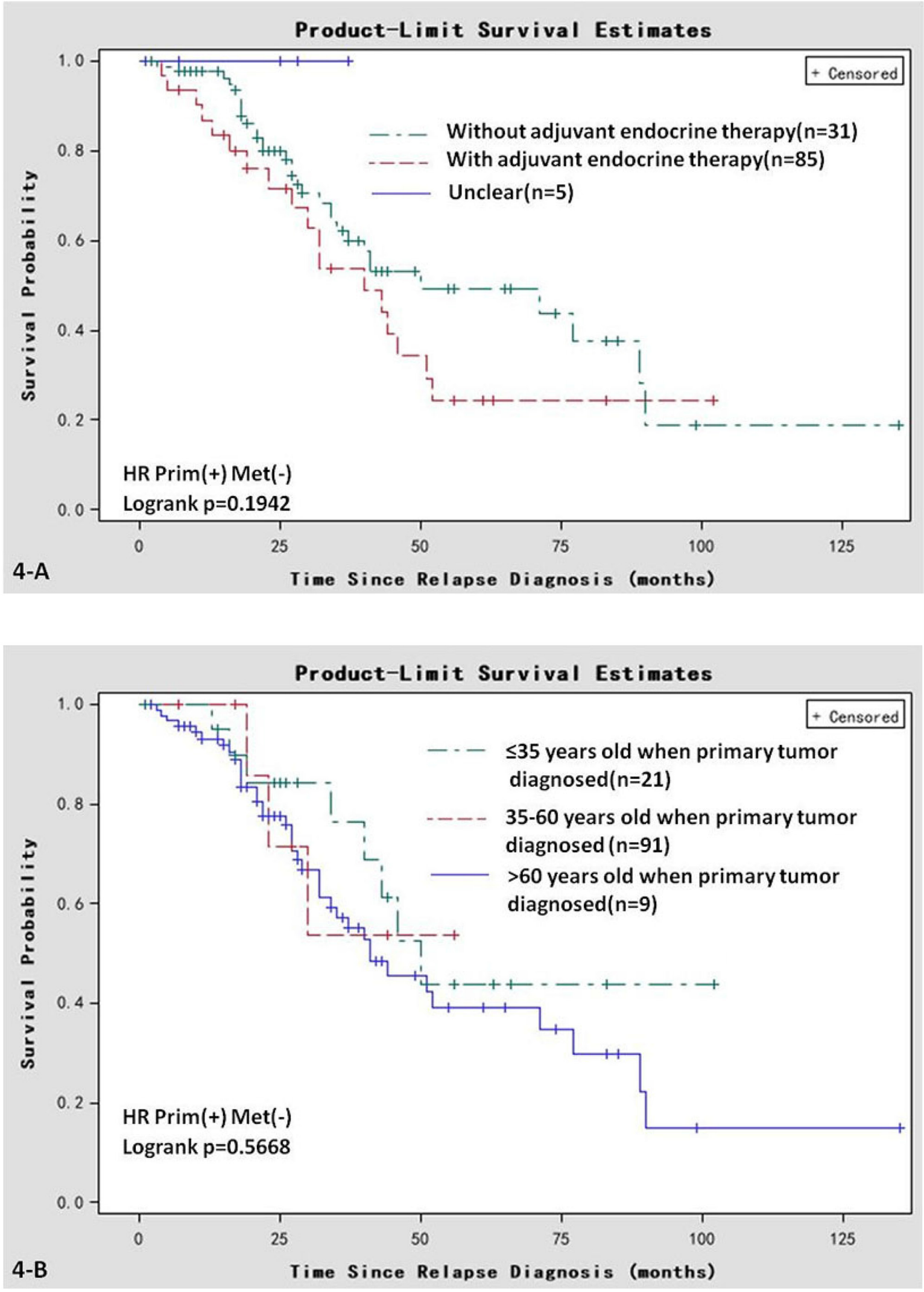

Figure 4: Univariate Kaplan-Meier analyses of post-recurrent survival of 121 patients in the $\mathrm{Prim}^{+} / \mathrm{Met}^{-}$subgroup. A. adjuvant endocrine therapy; B. age at primary diagnosis. (Continued) 

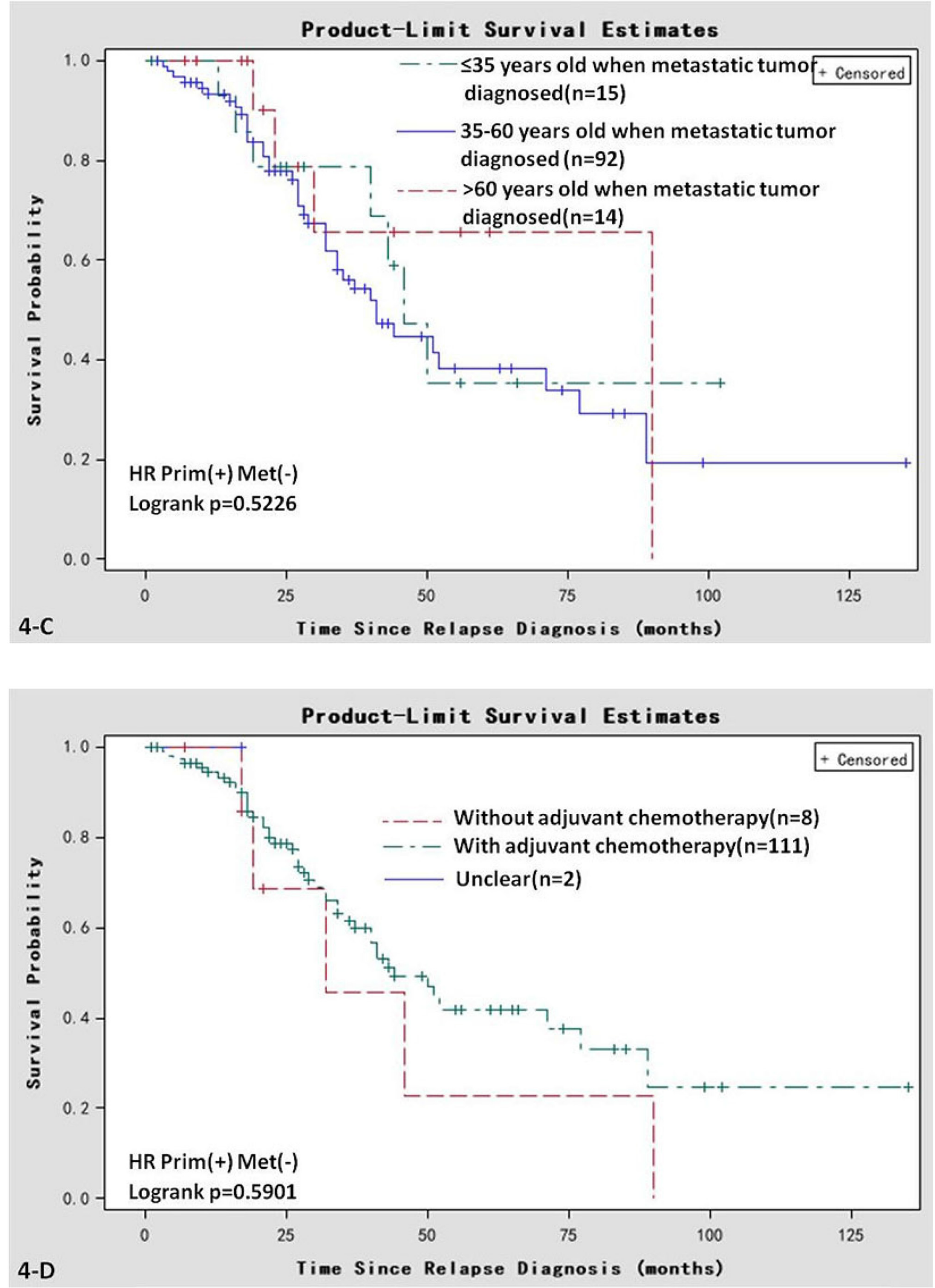

Figure 4: Univariate Kaplan-Meier analyses of post-recurrent survival of 121 patients in the $\mathrm{Prim}^{+} / \mathrm{Met}^{-}$subgroup. C. age at relapse diagnosis; D. adjuvant chemotherapy. (Continued) 

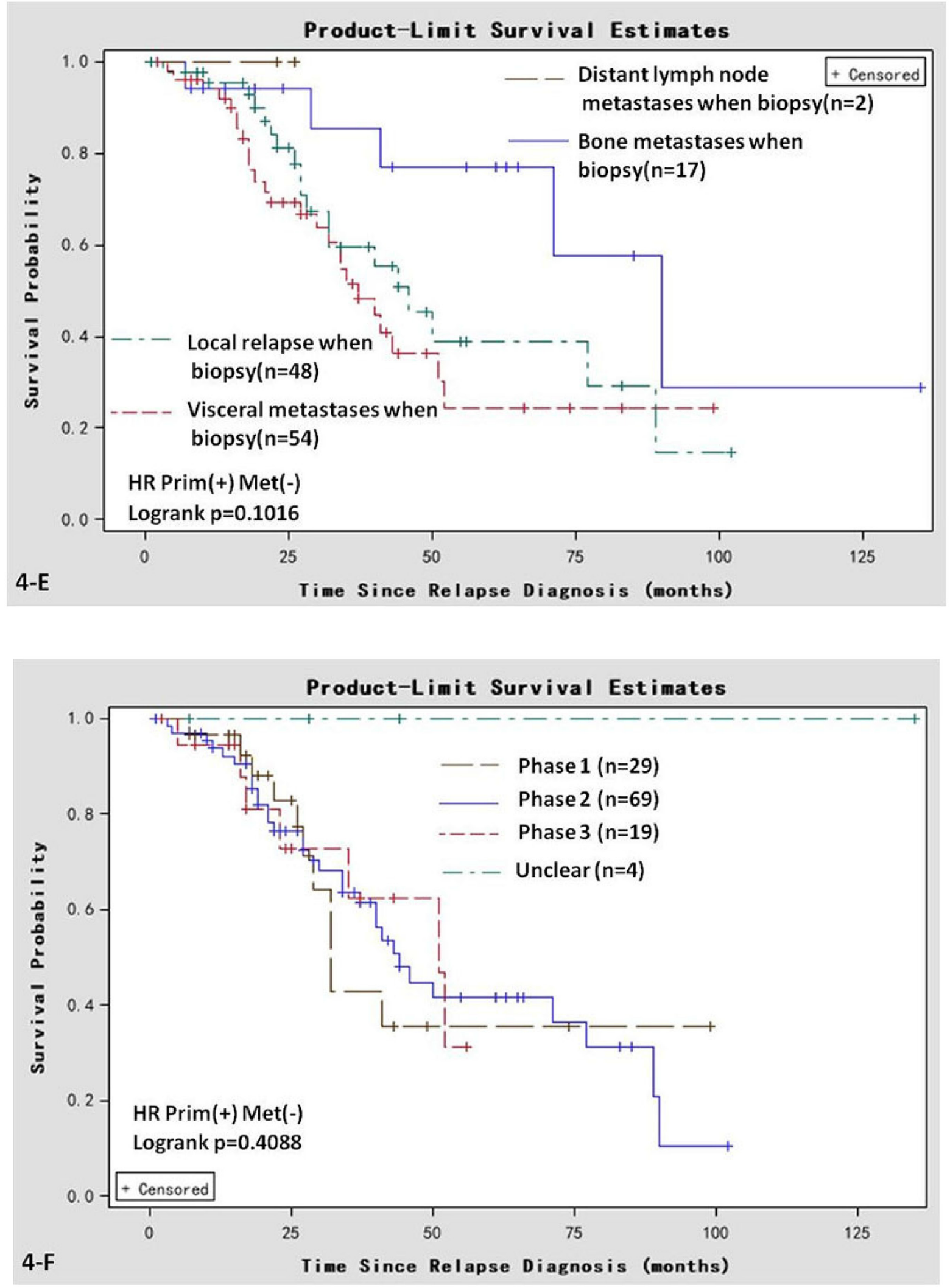

Figure 4: (Continued) Univariate Kaplan-Meier analyses of post-recurrent survival of 121 patients in the Prim $^{+} / \mathrm{Met}^{-}$ subgroup. E. metastatic sites; F. clinical phase. 
status are necessary in metastatic breast cancer. Salvage hormonal therapy is still worth trying in cases of positiveto-negative HR conversion.

\section{MATERIALS AND METHODS}

\section{Patients}

We conducted a single-center retrospective clinical study, initially collecting the clinical data of 3674 patients with invasive breast cancer who had been hospitalized sometime between 1 January 2002 and 1 April 2016 at Affiliated Hospital of Academy of Military Medical Sciences, Beijing, China.

The inclusion criteria were: pathologically confirmed breast cancer; metastatic disease biopsy; obtainable status of ER, PR, or both of primary and metastatic tumors; and receiving treatment provided by an oncology team that included an oncosurgeon, a medical oncologist, and a radiologist. Patients' clinicopathological information, including age, gender, date of invasive breast cancer diagnosis, date of metastatic diagnosis, location of distant metastasis, survival data, tumor pathological stage, adjuvant therapy regimen, and salvage therapy regimen was collected and recorded by the oncologists. Excluded from this study were patients without biopsy data on the metastasis with HR status, or patients with clinical stage IV breast cancer, bilateral primary breast cancer, contralateral breast metastasis, and those receiving neoadjuvant chemotherapy before biopsy.

The final analysis included 627 patients, all of whom had been biopsied at both the primary and paired metastatic sites. The academic and ethics committees of our hospital approved this retrospective study. All patients provided written informed consent before the biopsy.

\section{ER, PR, and HER-2 testing}

The primary and metastatic ER and PR statuses of each patient were required for this study and were obtained from the pathology reports. The ER and PR status were both evaluated using immunohistochemistry (IHC) as described previously [43]. Immunohistochemical analysis was carried out on full $4-\mu \mathrm{m}$ sections. For ER alpha $(\operatorname{ER} \alpha)$ and PR, the percentage of positively stained nuclei was estimated. Appropriate negative and positive controls were used throughout. A threshold $\geq 1 \%$ of stained nuclei was considered a positive status. Scoring of IHC slides was performed by 2 independent pathologists, blinded to other data in the paired samples. In primary tumor samples, the adequacy of staining was checked by comparison with normal breast parenchyma of the same patient.

We used 2 methods to assess tissue HER2 status in our study: IHC and fluorescence in situ hybridization (FISH). To evaluate HER2 overexpression, IHC staining of specimens was conducted using paraffin-embedded breast cancer tissues and polyclonal rabbit anti-human
HER2 oncoprotein. The latter targets the intracellular domain of HER2 protein. Tumors which scored 0 or $1+$ were designated HER2-negative; those that scored 3+ were considered HER2-positive. The tumors that were scored via IHC as 2+, were analyzed further by FISH using a PathVysion HER-2 DNA probe kit (Abbott Laboratories, Abbott Park, IL), in accordance with the manufacturer's recommended protocol. A FISH result was defined as positive when the HER2/cep17 ratio was $>2.2$.

\section{Statistical analysis}

Comparisons of ER and PR statuses between the primary tumor and paired metastatic sites were performed using McNemar's test. Overall survival (OS) was defined as the time from the date of the pathological diagnosis of primary breast cancer to the date of death or at the end of follow-up (1 May 2016). Post-recurrence survival was considered from the date of the pathological diagnosis of metastatic breast cancer to the date of death or at the end of follow-up (1 May 2016). The Kaplan-Meier analysis was applied to determine whether HR conversion could be a prognosticator of survival $[44,45]$. The risk of tumor-related death associated with ER and PR status was modeled using a multivariable Cox proportional hazard model. An arbitrary level of 5\% was used to indicate statistical significance. Our clinical data was analyzed using SAS version 9.2 statistical software.

\section{CONFLICTS OF INTEREST}

The authors have no conflicts of interest to declare.

\section{REFERENCES}

1. Siegel R, Naishadham D, Jemal A. Cancer statistics, 2012. CA Cancer J Clin. 2012; 62: 10-29. doi: 10.3322/ caac. 20138.

2. Siegel R, DeSantis C, Virgo K, Stein K, Mariotto A, Smith T, Cooper D, Gansler T, Lerro C, Fedewa S, Lin C, Leach C, Cannady RS, et al. Cancer treatment and survivorship statistics, 2012. CA Cancer J Clin. 2012; 62: 220-41. doi: $10.3322 /$ caac. 21149 .

3. Chia S, Bryce C, Gelmon K. The 2000 EBCTCG overview: a widening gap. Lancet. 2005; 365: 1665-6. doi: 10.1016/ s0140-6736(05)66524-5.

4. Davies C, Godwin J, Gray R, Clarke M, Cutter D, Darby S, McGale P, Pan HC, Taylor C, Wang YC, Dowsett M, Ingle J, Peto R. Relevance of breast cancer hormone receptors and other factors to the efficacy of adjuvant tamoxifen: patient-level meta-analysis of randomised trials. Lancet. 2011; 378: 771-84. doi: 10.1016/ s0140-6736(11)60993-8.

5. Dowsett M, Folkerd E. Deficits in plasma oestradiol measurement in studies and management of breast cancer. Breast Cancer Res. 2005; 7: 1-4. doi: 10.1186/bcr960. 
6. Berry DA, Cronin KA, Plevritis SK, Fryback DG, Clarke L, Zelen M, Mandelblatt JS, Yakovlev AY, Habbema JD, Feuer EJ. Effect of screening and adjuvant therapy on mortality from breast cancer. N Engl J Med. 2005; 353: 1784-92. doi: 10.1056/NEJMoa050518.

7. Coates AS, Keshaviah A, Thurlimann B, Mouridsen H, Mauriac L, Forbes JF, Paridaens R, Castiglione-Gertsch M, Gelber RD, Colleoni M, Lang I, Del Mastro L, Smith I, et al. Five years of letrozole compared with tamoxifen as initial adjuvant therapy for postmenopausal women with endocrine-responsive early breast cancer: update of study BIG 1-98. Journal of clinical oncology. 2007; 25: 486-92. doi: 10.1200/JCO.2006.08.8617.

8. Howell A, Cuzick J, Baum M, Buzdar A, Dowsett M, Forbes JF, Hoctin-Boes G, Houghton J, Locker GY, Tobias JS. Results of the ATAC (Arimidex, Tamoxifen, Alone or in Combination) trial after completion of 5 years' adjuvant treatment for breast cancer. Lancet. 2005; 365: 60-2. doi: 10.1016/S0140-6736(04)17666-6.

9. Arslan C, Sari E, Aksoy S, Altundag K. Variation in hormone receptor and HER-2 status between primary and metastatic breast cancer: review of the literature. Expert Opin Ther Targets. 2011; 15: 21-30. doi: 10.1517/14656566.2011.537260.

10. Amir E, Miller N, Geddie W, Freedman O, Kassam F, Simmons C, Oldfield M, Dranitsaris G, Tomlinson G, Laupacis A, Tannock IF, Clemons M. Prospective study evaluating the impact of tissue confirmation of metastatic disease in patients with breast cancer. J Clin Oncol. 2012; 30: 587-92. doi: 10.1200/jco.2010.33.5232.

11. Brennan MJ, Donegan WL, Appleby DE. The variability of estrogen receptors in metastatic breast cancer. Am J Surg. 1979; 137: 260-2.

12. Dieci MV, Barbieri E, Piacentini F, Ficarra G, Bettelli S, Dominici M, Conte PF, Guarneri V. Discordance in receptor status between primary and recurrent breast cancer has a prognostic impact: a single-institution analysis. Ann Oncol. 2013; 24: 101-8. doi: 10.1093/annonc/mds248.

13. Guarneri V, Giovannelli S, Ficarra G, Bettelli S, Maiorana A, Piacentini F, Barbieri E, Dieci MV, D’Amico R, Jovic G, Conte P. Comparison of HER-2 and hormone receptor expression in primary breast cancers and asynchronous paired metastases: impact on patient management. Oncologist. 2008; 13: 838-44. doi: 10.1634/ theoncologist.2008-0048.

14. Hirata T, Shimizu C, Yonemori K, Hirakawa A, Kouno T, Tamura K, Ando M, Katsumata N, Fujiwara Y. Change in the hormone receptor status following administration of neoadjuvant chemotherapy and its impact on the long-term outcome in patients with primary breast cancer. Br J Cancer. 2009; 101: 1529-36. doi: 10.1038/sj.bjc.6605360.

15. Hoefnagel LD, van de Vijver MJ, van Slooten HJ, Wesseling P, Wesseling J, Westenend PJ, Bart J, Seldenrijk CA, Nagtegaal ID, Oudejans J, van der Valk P, van der Groep P, de Vries EG, et al. Receptor conversion in distant breast cancer metastases. Breast Cancer Res. 2010; 12: R75. doi: $10.1186 /$ bcr2645.

16. Holdaway IM, Bowditch JV. Variation in receptor status between primary and metastatic breast cancer. Cancer. 1983; 52: 479-85.

17. Thompson AM, Jordan LB, Quinlan P, Anderson E, Skene A, Dewar JA, Purdie CA. Prospective comparison of switches in biomarker status between primary and recurrent breast cancer: the Breast Recurrence In Tissues Study (BRITS). Breast Cancer Res. 2010; 12: R92. doi: 10.1186/ bcr2771.

18. Kroigard AB, Larsen MJ, Thomassen M, Kruse TA. Molecular Concordance Between Primary Breast Cancer and Matched Metastases. Breast J. 2016. doi: 10.1111/ tbj. 12596.

19. Kimbung S, Kovacs A, Danielsson A, Bendahl PO, Lovgren K, Frostvik Stolt M, Tobin NP, Lindstrom L, Bergh J, Einbeigi Z, Ferno M, Hatschek T, Hedenfalk I. Contrasting breast cancer molecular subtypes across serial tumor progression stages: biological and prognostic implications. Oncotarget. 2015; 6: 33306-18. doi: 10.18632/ oncotarget.5089.

20. Kalinsky K, Mayer JA, Xu X, Pham T, Wong KL, Villarin E, Pircher TJ, Brown M, Maurer MA, Bischoff FZ. Correlation of hormone receptor status between circulating tumor cells, primary tumor, and metastasis in breast cancer patients. Clin Transl Oncol. 2015; 17: 539-46. doi: 10.1007/ s12094-015-1275-1.

21. Amir E, Miller N, Geddie W, Freedman O, Kassam F, Simmons C, Oldfield M, Dranitsaris G, Tomlinson G, Laupacis A, Tannock IF, Clemons M. Prospective study evaluating the impact of tissue confirmation of metastatic disease in patients with breast cancer. Journal of clinical oncology. 2012; 30: 587-92. doi: 10.1200/ JCO.2010.33.5232.

22. Thompson AM, Jordan LB, Quinlan P, Anderson E, Skene A, Dewar JA, Purdie CA. Prospective comparison of switches in biomarker status between primary and recurrent breast cancer: the Breast Recurrence In Tissues Study (BRITS). Breast cancer research. 2010; 12: R92. doi: 10.1186/bcr2771.

23. Bartlett JM, Brookes CL, Robson T, van de Velde CJ, Billingham LJ, Campbell FM, Grant M, Hasenburg A, Hille ET, Kay C, Kieback DG, Putter H, Markopoulos $\mathrm{C}$, et al. Estrogen receptor and progesterone receptor as predictive biomarkers of response to endocrine therapy: a prospectively powered pathology study in the Tamoxifen and Exemestane Adjuvant Multinational trial. J Clin Oncol. 2011; 29: 1531-8. doi: 10.1200/jco.2010.30.3677.

24. Chang J, Clark GM, Allred DC, Mohsin S, Chamness G, Elledge RM. Survival of patients with metastatic breast carcinoma: importance of prognostic markers of the primary tumor. Cancer. 2003; 97: 545-53. doi: 10.1002/cncr.11083.

25. Liedtke C, Broglio K, Moulder S, Hsu L, Kau SW, Symmans WF, Albarracin C, Meric-Bernstam F, Woodward 
W, Theriault RL, Kiesel L, Hortobagyi GN, Pusztai L, et al. Prognostic impact of discordance between triple-receptor measurements in primary and recurrent breast cancer. Annals of oncology. 2009; 20: 1953-8. doi: 10.1093/ annonc/mdp263.

26. Dieci MV, Barbieri E, Piacentini F, Ficarra G, Bettelli S, Dominici M, Conte PF, Guarneri V. Discordance in receptor status between primary and recurrent breast cancer has a prognostic impact: a single-Institution analysis. Annals of oncology. 2012. doi: 10.1093/annonc/mds248.

27. Brennan MJ, Donegan WL, Appleby DE. The variability of estrogen receptors in metastatic breast cancer. American journal of surgery. 1979; 137: 260-2.

28. Lower EE, Glass EL, Bradley DA, Blau R, Heffelfinger $\mathrm{S}$. Impact of metastatic estrogen receptor and progesterone receptor status on survival. Breast Cancer Research and Treatment. 2005; 90: 65-70. doi: 10.1007/ s10549-004-2756-z.

29. Curigliano G, Bagnardi V, Viale G, Fumagalli L, Rotmensz N, Aurilio G, Locatelli M, Pruneri G, Giudici S, Bellomi M, Della Vigna P, Monfardini L, Orsi F, et al. Should liver metastases of breast cancer be biopsied to improve treatment choice? Annals of oncology. 2011; 22: 2227-33. doi: 10.1093/annonc/mdq751.

30. Diaz LK, Sneige N. Estrogen receptor analysis for breast cancer: current issues and keys to increasing testing accuracy. Advances in anatomic pathology. 2005; 12: 10-9.

31. Guarneri V, Giovannelli S, Ficarra G, Bettelli S, Maiorana A, Piacentini F, Barbieri E, Dieci MV, D’Amico R, Jovic G, Conte P. Comparison of HER-2 and hormone receptor expression in primary breast cancers and asynchronous paired metastases: impact on patient management. The oncologist. 2008; 13: 838-44. doi: 10.1634/ theoncologist.2008-0048.

32. Hirata T, Shimizu C, Yonemori K, Hirakawa A, Kouno T, Tamura K, Ando M, Katsumata N, Fujiwara Y. Change in the hormone receptor status following administration of neoadjuvant chemotherapy and its impact on the long-term outcome in patients with primary breast cancer. British Journal of Cancer. 2009; 101: 1529-36. doi: 10.1038/ sj.bjc. 6605360 .

33. Hoefnagel LD, Moelans CB, Meijer SL, van Slooten HJ, Wesseling P, Wesseling J, Westenend PJ, Bart J, Seldenrijk CA, Nagtegaal ID, Oudejans J, van der Valk P, van Gils CH, et al. Prognostic value of estrogen receptor alpha and progesterone receptor conversion in distant breast cancer metastases. Cancer. 2012; 118: 4929-35. doi: 10.1002/cncr.27518.

34. Hoefnagel LD, van de Vijver MJ, van Slooten HJ, Wesseling P, Wesseling J, Westenend PJ, Bart J, Seldenrijk CA, Nagtegaal ID, Oudejans J, van der Valk P, van der Groep P, de Vries EG, et al. Receptor conversion in distant breast cancer metastases. Breast cancer research. 2010; 12: R75. doi: 10.1186/bcr2645.
35. Idirisinghe PK, Thike AA, Cheok PY, Tse GM, Lui PC, Fook-Chong S, Wong NS, Tan PH. Hormone receptor and c-ERBB2 status in distant metastatic and locally recurrent breast cancer. Pathologic correlations and clinical significance. American Journal of Clinical Pathology. 2010; 133: 416-29. doi: 10.1309/ AJCPJ57FLLJRXKPV.

36. Simmons C, Miller N, Geddie W, Gianfelice D, Oldfield M, Dranitsaris G, Clemons MJ. Does confirmatory tumor biopsy alter the management of breast cancer patients with distant metastases? Annals of oncology. 2009; 20: 1499504. doi: 10.1093/annonc/mdp028.

37. Wu JM, Fackler MJ, Halushka MK, Molavi DW, Taylor ME, Teo WW, Griffin C, Fetting J, Davidson NE, De Marzo AM, Hicks JL, Chitale D, Ladanyi M, et al. Heterogeneity of breast cancer metastases: comparison of therapeutic target expression and promoter methylation between primary tumors and their multifocal metastases. Clinical cancer research. 2008; 14: 1938-46. doi: 10.1158/10780432.CCR-07-4082.

38. Tan QX, Qin QH, Yang WP, Lian B, Wei CY. Prognostic value of hormone receptor status conversion following neoadjuvant chemotherapy in a series of operable breast cancer patients. Int J Clin Exp Pathol. 2014; 7: 4086-94.

39. Lindstrom LS, Karlsson E, Wilking UM, Johansson U, Hartman J, Lidbrink EK, Hatschek T, Skoog L, Bergh J. Clinically used breast cancer markers such as estrogen receptor, progesterone receptor, and human epidermal growth factor receptor 2 are unstable throughout tumor progression. Journal of clinical oncology. 2012; 30: 26018. doi: 10.1200/JCO.2011.37.2482.

40. Lower EE, Blau R, Gazder P, Stahl DL. The effect of estrogen usage on the subsequent hormone receptor status of primary breast cancer. Breast Cancer Research and Treatment. 1999; 58: 205-11.

41. Liedtke C, Broglio K, Moulder S, Hsu L, Kau SW, Symmans WF, Albarracin C, Meric-Bernstam F, Woodward W, Theriault RL, Kiesel L, Hortobagyi GN, Pusztai L, et al. Prognostic impact of discordance between triple-receptor measurements in primary and recurrent breast cancer. Ann Oncol. 2009; 20: 1953-8. doi: 10.1093/ annonc/mdp263.

42. Lindstrom LS, Karlsson E, Wilking UM, Johansson U, Hartman J, Lidbrink EK, Hatschek T, Skoog L, Bergh J. Clinically used breast cancer markers such as estrogen receptor, progesterone receptor, and human epidermal growth factor receptor 2 are unstable throughout tumor progression. J Clin Oncol. 2012; 30: 2601-8. doi: 10.1200/ jco.2011.37.2482.

43. Lower EE, Blau R, Gazder P, Stahl DL. The effect of estrogen usage on the subsequent hormone receptor status of primary breast cancer. Breast Cancer Res Treat. 1999; 58: 205-11. 
44. Dinh P, Zhou XH. Nonparametric statistical methods for cost-effectiveness analyses. Biometrics. 2006; 62: 576-88. doi: 10.1111/j.1541-0420.2006.00509.x.
45. Gerald KB. Nonparametric statistical methods. Nurse anesthesia. 1991; 2: 93-5. 